\title{
Face-to-Face EML Transceiver Tandem for Full-Duplex Analogue Radio-over-Air
}

\author{
Bernhard Schrenk, Member, IEEE, Dinka Milovančev, Member, IEEE, Nemanja Vokić, Member, \\ IEEE, Hannes Hübel, and Fotini Karinou, Member, IEEE
}

\begin{abstract}
An analogue coherent-optical free-space link for local cloud-based radio access networks is proposed and experimentally demonstrated. The adoption of a single externally modulated laser as optical transmitter and coherent homodyne receiver at either link end guarantees conceptual simplicity for the opto-electronic sub-systems of this optical fronthaul. The realization of full-duplex signal transmission over a single, laserbased transceiver device further allows to off-load the directional split in the optically fronthauled radio signal chain to the radiofrequency domain. We prove this concept for an in-door link and show that bidirectional transmission of orthogonally frequency division multiplexed radio signals with 64-point quadrature amplitude modulated formats is possible over an optical budget of $21.3 \mathrm{~dB}$, or over an estimated reach of $\sim 100 \mathrm{~m}$ in case of bad atmospheric conditions. A small penalty of less than $1 \%$ in terms of error vector magnitude compared to a direct-detection receiver confirms the correct operation of the low-complexity coherent homodyne detector, even though no digital signal processing functions are applied for the purpose of signal recovery. Continuous long-term measurements including Ethernet payloads confirm the stability of the free-space optical link architecture, for which a small fraction of less than $1 \%$ has shown an excursion in reception penalty.
\end{abstract}

Index Terms-Optical communication terminals, Free-space optical communication, Optical signal detection, Mobile fronthaul

\section{INTRODUCTION}

$\mathrm{T}$ HE ongoing densification of wireless networks and the adoption of mobile fronthauling for the purpose of cloudbased radio signal processing render optical communication technology as an important pillar of $5 \mathrm{G}$ infrastructure. In such cloud-based radio access networks (C-RAN), the signals of many remote radio heads $(\mathrm{RRH})$ are jointly processed in a datacenter warehouse where computational resources of baseband units (BBU) can be effectively pooled [1]. However, the strict latency requirements of time-sensitive applications

Manuscript received November 11, 2019. This work was supported in part by the European Research Council (ERC) under the European Union's Horizon 2020 research and innovation programme (grant agreement No 804769).

B. Schrenk, D. Milovancev, N. Vokic and H. Hübel are with the Austrian Institute of Technology, Center for Digital Safety\&Security, Giefinggasse 4, 1210 Vienna, Austria (phone: +43 50550-4131; fax: -4150; e-mail: bernhard.schrenk@ait.ac.at).

F. Karinou is with Microsoft Research Ltd, Cambridge, CB1 2FB, United Kingdom (e-mail: fotini.karinou@microsoft.com).

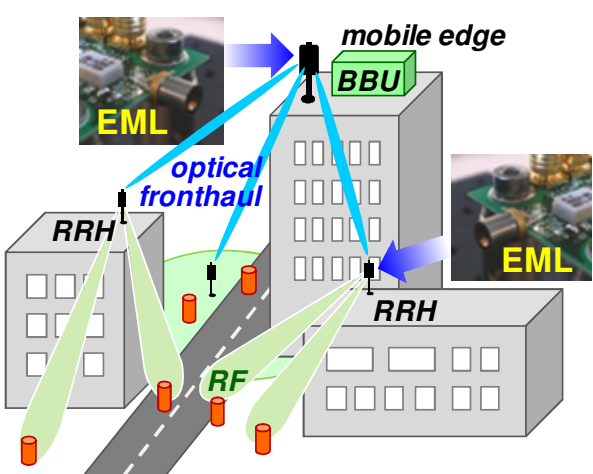

Fig. 1. Local C-RAN building on a free-space optical fronthaul with face-toface EML arrangement.

require mobile edge computing close to the end-user [2], which suggests the deployment of local C-RANs.

Mobile fronthauling can be in principle supported by radio frequency (RF) communications at elevated carrier frequencies. Despite the progress in mm-wave technology [3], the delivery of multi-band radio is often strongly limited by the system bandwidth and propagation effects. For example, self-interference and operation at low heights may cause frequent interruptions for mm-wave links.

In light of this, the beneficial properties of broadband optics allow for transparent antenna remoting, which is mostly accomplished over deployed fiber [4]. The bandwidth of fiberoptic links can be exploited by digitizing the radio signal and transmitting it as binary signal [5,6]. However, in order to retain a low complexity during optical signal relay, analogueto-digital conversion should be omitted. Instead, analogue optical radio signal transmission in combination with linear opto-electronic converters [7,8] achieves transparent fronthauling and thus guarantees a cost- and energy-effective method.

Optical fiber is nevertheless a scarce resource and might not be available in many deployment scenarios. Therefore, freespace optics [9-11] can be an alternative solution for local optical line-of-sight fronthauling (Fig. 1). Unlike point-tomultipoint fiber networks where complex opto-electronic transceivers can be cost-shared at the head-end of the link, such free-space point-to-point links require conceptually simple and ideally analogue transceivers at both, RRH and BBU sites.

In this work, we experimentally demonstrate full-duplex 

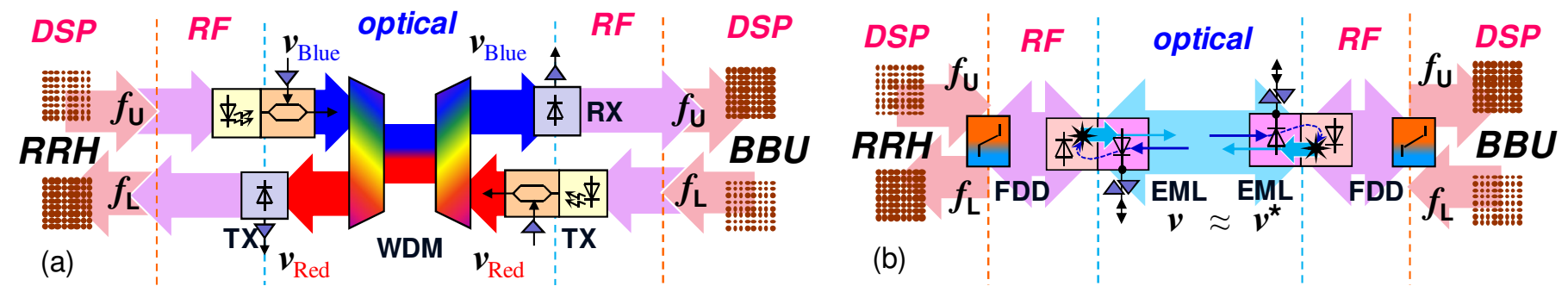

Fig. 2. Signal chain for (a) traditional optical link with dedicated transmitter and receiver sub-system and directional split in the optical domain, exploiting two wavelengths $v_{\text {Blue }}$ and $v_{\text {Red }}$, and (b) proposed link with face-to-face EML transceiver arrangement and directional signal split off-loaded to the RF domain, which implements two frequency bands $f_{\mathrm{U}}, f_{\mathrm{L}}$.

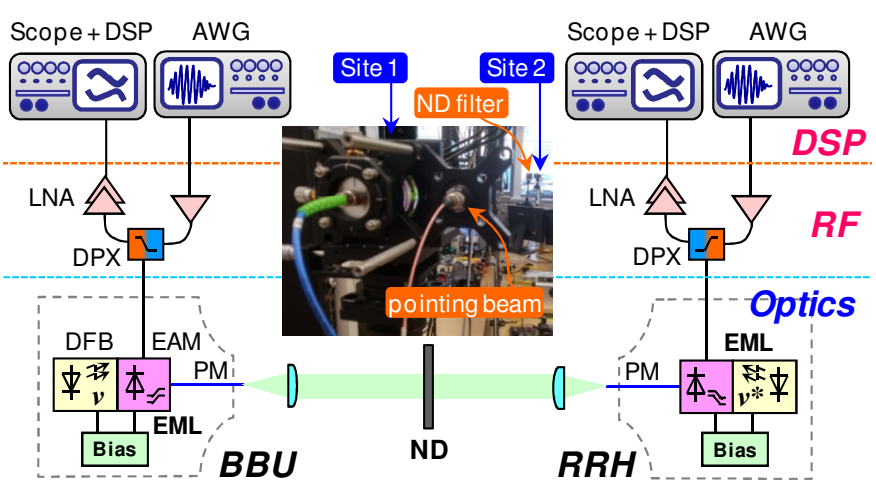

Fig. 3. Experimental setup of the free-space optical lab link.

analogue radio-over-air transmission using an externally modulated laser (EML) as both, transmitter and receiver. This low-complexity transceiver is employed at head- and tail-end sites of an optical fronthaul link. We show bidirectional orthogonal frequency division multiplexed (OFDM) transmission loaded with 64-point quadrature amplitude modulation (QAM) over an optical budget of $21.3 \mathrm{~dB}$. Compared to a half-duplex receiver based on a PIN photodiode, a small $<1 \%$ penalty in error vector magnitude (EVM) is experienced for the EML-based transceiver pair in full-duplex mode. In addition to the static measurements on this analogue optical radio pipe, as previously introduced in brevity [12], long-term measurements including Ethernetbased data transmission and high-definition video streaming are conducted to investigate the stability of this face-to-face EML transceiver configuration.

This paper is organized as follows. Section II introduces the transparent analogue radio-over-air link. Section III presents the experimental settings, while Section IV discusses the transmission performance. Section V evaluates the long-term performance of the face-to-face transceiver arrangement. Finally, Section VI concludes the work.

\section{Fronthaul Based on EML Transceiver Pair}

State-of-play optical transmission systems build on dedicated transmitter and receiver sub-systems that are laid out for the sole purpose of opto-electronic conversion from the optical to the RF domain, or vice versa. The directional split, which enables bidirectional use of the channel, is commonly implemented in the optical domain by means of duplexing, for which wavelength division multiplexing (WDM) is typically applied. The corresponding signal chain for such a full-duplex transmission link is illustrated in Fig. 2(a).

In the present work, we build on a versatile transceiver that can perform both functions, transmission and reception. Moreover, the employed transceiver performs these functions simultaneously without doubling the required opto-electronic components. A simple device such as an EML suits these requirements, as we have recently demonstrated [13]. Its electro-absorption modulator (EAM) can be used to modulate an RF signal on an optical carrier provided by the distributed feedback (DFB) laser section. Alternatively, the EAM can serve as photodiode to absorb an optical signal that is delivered to the EML. The emission from the DFB section can be exploited as a local oscillator (LO). The detuning between local emission and injected signal determines the electrical frequency to which the input signal is converted. In this way, photonic up-conversion of a baseband signal can be accomplished [14]. For the particular case that the DFB section is biased to emit nearly at the same optical frequency as the incident signal, the external optical feed to the EML will, in case it is strong enough, lock the DFB laser so that two fields with the same optical frequency beat at the EAM. The requirement to do so is a frequency detuning between the two optical sources within the injection locking range, which for the employed EMLs typically is $200 \mathrm{MHz}$ for an injected power of $-30 \mathrm{dBm}$ [13]. Homodyne signal detection can thus be realized in an analogue coherent receiver implementation that does not require additional digital signal processing (DSP) functions to correct a frequency mismatch between the local oscillator and received optical signal [15].

Both fundamental functions, transmission and coherent reception, can be facilitated simultaneously, which leads to a transceiver implementation with a single fiber and a single RF port. This results in a very low physical-layer complexity and cost reductions in component assembly and packaging for the BBU and RRH optics. The signal flow for such a technologically lean link is reported in Fig. 2(b). The directional split is advantageously shifted from the optical domain to the RF domain. Duplexing is now applied at the frequency rather than on the wavelength level and serves the prerequisite that crosstalk among the transmitted and received signal is minimized through non-overlapping spectra. Frequency division duplex (FDD) of the radio signals at the two carrier frequencies $f_{\mathrm{U}}$ and $f_{\mathrm{L}}$ is employed for this purpose 


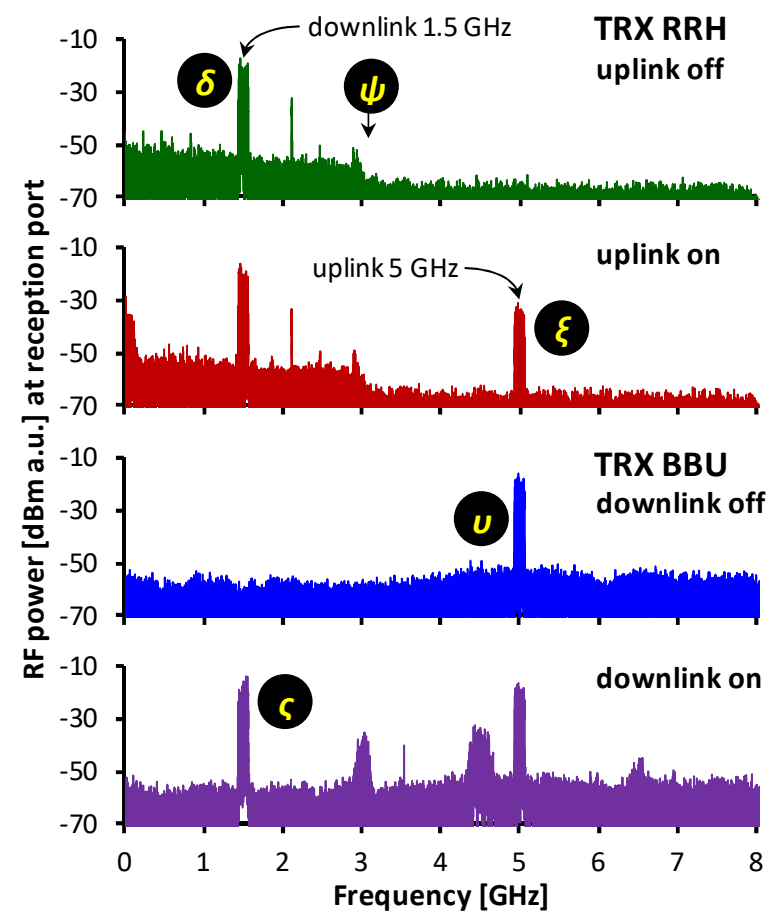

Fig. 4. Received RF signal spectra at the RRH and the BBU for half- and full-duplex down-/uplink radio transmission. The spectra are characterized by the down- $(\delta)$ and upstream $(v)$, the respective crosstalk notes $\varsigma$ and $\xi$ and the edge frequency $\psi$ of the duplexer.

of simultaneous up-/downlink transmission. FDD is foreseen in 5G radio systems with a paired spectrum [16]. Alternatively, a common carrier frequency could be used in combination with a frequency-agnostic RF circulator with high port isolation or time division duplexing.

It shall be noted that the spectral selectivity that is inherently provided through the coherent detection process is not exploited since the present work aims at a point-to-point link. However, it supports the robustness of the free-space link to residual near infra-red components of the sunlight or other luminaries installed in the vicinity of the BBU and RRH transceivers. In addition the reception sensitivity is improved compared to direct-detection systems. It shall further be pointed out that polarization management, which is typically mandatory for coherent reception, can be omitted as the freespace channel is non-birefringent.

Compared to our previous work [17], the same transceiver methodology is employed, yet at both, head- and tail-end of the link. Through that a symmetrical optical pipe with lowcost transceivers at both sites is yielded, which greatly simplifies the link architecture. In particular, [17] used an optically isolated dual-feeder fiber which is common in optical access, but may not apply to other communication segments. By using the same low-complexity transceiver at both end points of the link, as endeavored in the present paper, costeffective deployment is enabled, even in point-to-point applications where no cost-sharing applies. However, an investigation on the stability of such a link design is required, since no optical isolation is now employed anymore.

\section{EXPERIMENTAL FREE-SPACE OPTICAL RADIO LINK}

The proposed concept was evaluated in an in-door lab environment, for which the experimental in-door link setup is presented in Fig. 3.

\section{A. Optical Plane}

A non-commercial transistor-outline (TO) EML with integrated micro-cooler and an emission wavelength of 1547.7 $\mathrm{nm}$ is employed as optical transceiver at BBU and RRH. The EMLs are coupled to a telescope at each end of the link through a polarization-maintaining (PM) patchcord. A 2-inch collimation lens ensures good coupling between the two telescopes, which were spaced by a distance of 5 meters. The optical budget between the fiber ports of the EML at BBU and RRH was $19.3 \mathrm{~dB}$. A neutral density (ND) filter has been inserted between the telescopes to further emulate an increased free-space path loss as it would apply for an extended line-of-sight link reach.

In order to perform transparent translation of the radio signals between optical and RF domain through coherent homodyne reception, the emission frequencies $v, v^{*}$ of both EMLs are tuned through temperature and DFB bias current setting so that one of the emission frequencies is falling within the injection locking range of the other. Unlike the EAM bias, which is optimized according to full-duplex transmission, as will be discussed shortly, the DFB bias current is set irrespectively of the duplex mode.

\section{B. RF Plane}

The EAM as opto-electronic converter connects the optical to the RF plane. The FDD scheme and thus the functional split is implemented through a duplexer (DPX). Its edge frequency of $3.1 \mathrm{GHz}$ splits the lower $\left(f_{\mathrm{L}}\right)$ and upper $\left(f_{\mathrm{U}}\right)$ frequency bands for bidirectional radio signal transmission in down- and uplink direction, respectively. A low-noise amplifier (LNA) is used for signal conditioning after coherent optical reception. This is sub-optimal in terms of noise and bandwidth performance; however, it allows to prove the concept in combination with TO-can EMLs for which no external co-integration with a transimpedance amplifier (TIA) is possible.

\section{DSP Plane}

The radio signals have been generated with an arbitrary waveform generator (AWG). OFDM signals with 128 subcarriers over a bandwidth of $125 \mathrm{MHz}$ have been used in down- and uplink direction. 64-QAM was loaded to all subcarriers. The OFDM carrier frequencies were $f_{\mathrm{L}}=1.5 \mathrm{GHz}$ for the downlink and $f_{\mathrm{U}}=5 \mathrm{GHz}$ for the uplink signal. The reception performance is evaluated through acquisition of the received radio signals with a real-time oscilloscope and subsequent off-line signal demodulation and EVM estimation. There have been no further DSP functions adopted to compensate channel or reception impairments.

It shall be stressed that radio signal transmission in millimeter-wave bands allows for higher signal bandwidths that exceed $1 \mathrm{GHz}$ [18]. A high-bandwidth radio signal can be supported by the large electro-optic EAM bandwidth, 

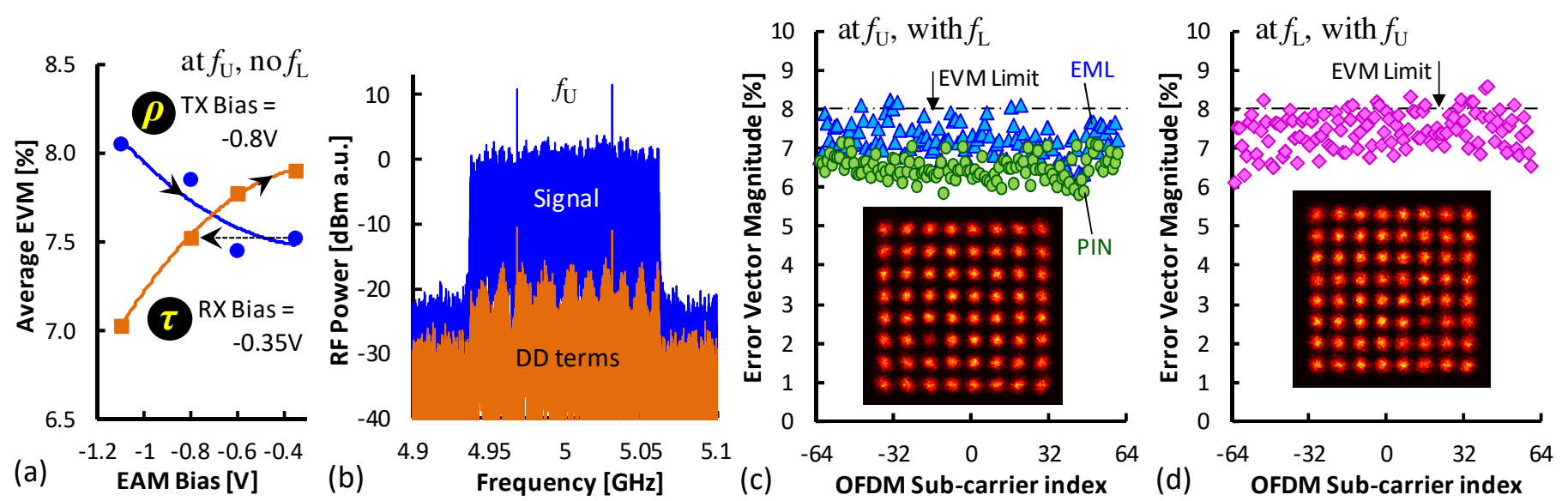

Fig. 5. (a) Reception performance during EAM bias optimization when varying the bias at the receiving $(\rho)$ and transmitting $(\tau)$ EML. (b) Received uplink radio signal spectrum under coherent and direct detection. EVM under full-duplex operation for (c) the uplink and (d) the downlink channel.

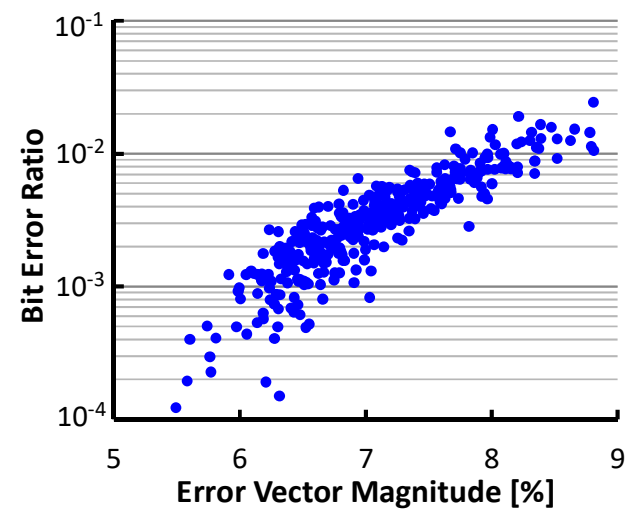

Fig. 6. BER obtained in correspondence with the estimated EVM.

provided that the EML device is amenable for co-integration with a TIA-based front-end at low parasitic capacitance. Since this was not the case for the present TO-can EML, the radio signal bandwidth has been chosen with $125 \mathrm{MHz}$.

\section{Full-Duplex Radio Signal TRANSMission}

\section{A. Spectral Integrity}

The received radio signal spectra after coherent homodyne detection are shown in Fig. 4 at the RRH and BBU receiver. At both full-duplex operated EML transceivers, analogue coherent reception is providing a correct signal translation during the conversion between the RF and the optical domain, even without assistance through DSP. This is evidenced by the clearly delimited OFDM signal spectra for both, down- $(\delta)$ and uplink $(v)$, which confirm the correct homodyne operation. Otherwise, coherent intradyne signal reception would wash out these spectra due to a residual and time-dependent frequency offset.

The excellent signal integrity of the downlink OFDM signal $\delta$, transmitted from the BBU at $f_{\mathrm{L}}$, is not affected when the uplink transmitter at the RRH is activated. As Fig. 4 shows, the finite RF isolation at the DPX leads to a crosstalk $\xi$ at the uplink band at $f_{\mathrm{U}}$. However, this out-of-band crosstalk note does not lead to increased noise below the edge frequency $\psi$ of the DPX.

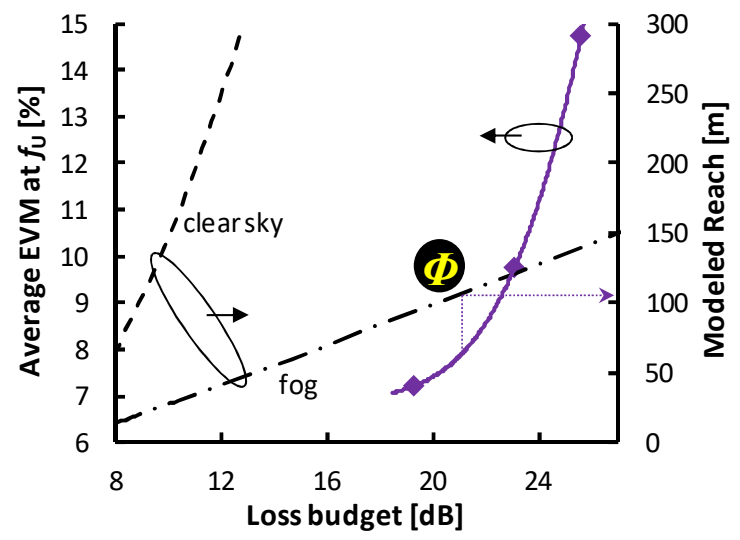

Fig. 7. Reception performance as function of the loss budget and estimated link reach that would result from the compatible loss budget $\Phi$.

Similar conditions apply for the uplink, which is received at the BBU. An activated downlink transmitter, emitting at $f_{\mathrm{L}}$, causes a crosstalk signal $\varsigma$ in the lower frequency band. Higher-order products appear due to non-linearity at 3 and 4.5 $\mathrm{GHz}$; however, the received uplink OFDM signal $v$ is not affected.

\section{B. Optimal Transceiver Bias}

The radio signal transmission performance over the freespace optical fronthaul has been assessed in terms of EVM measurements. In order to perform the compelling task of fullduplex signal transmission by using not more than a single EML, its EAM bias point has to be first optimized to simultaneously accommodate transmission and reception. While the bias of the transmitting EAM determines the intensity extinction and the magnitude of generated non-linear products, the bias of the receiving EAM determines the signal absorption and locking range. Figure 5(a) shows the average EVM for 64-QAM OFDM reception at $f_{\mathrm{U}}$ at the BBU receiver in half-duplex mode, meaning that there is no concurrent radio signal transmission at $f_{\mathrm{L}}$.

To investigate the impact of the bias point setting, the bias of the transmitting EML at the RRH is first kept constant at $-0.8 \mathrm{~V}$ while the bias of the receiving EML at the BBU is varied (curve $\rho$ ). At this transmitter bias, an intensity 

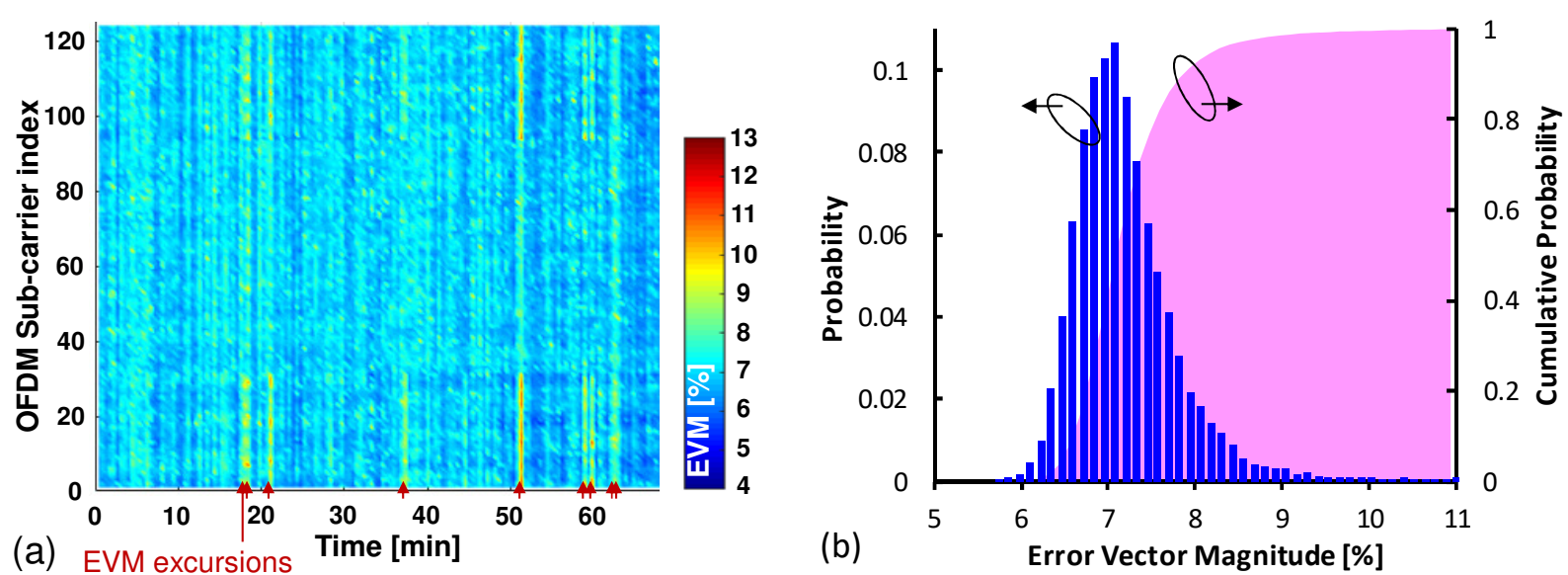

Fig. 8. Long-term reception performance. (a) EVM measurements for the uplink channel under full-duplex operation and (b) corresponding EVM histogram.

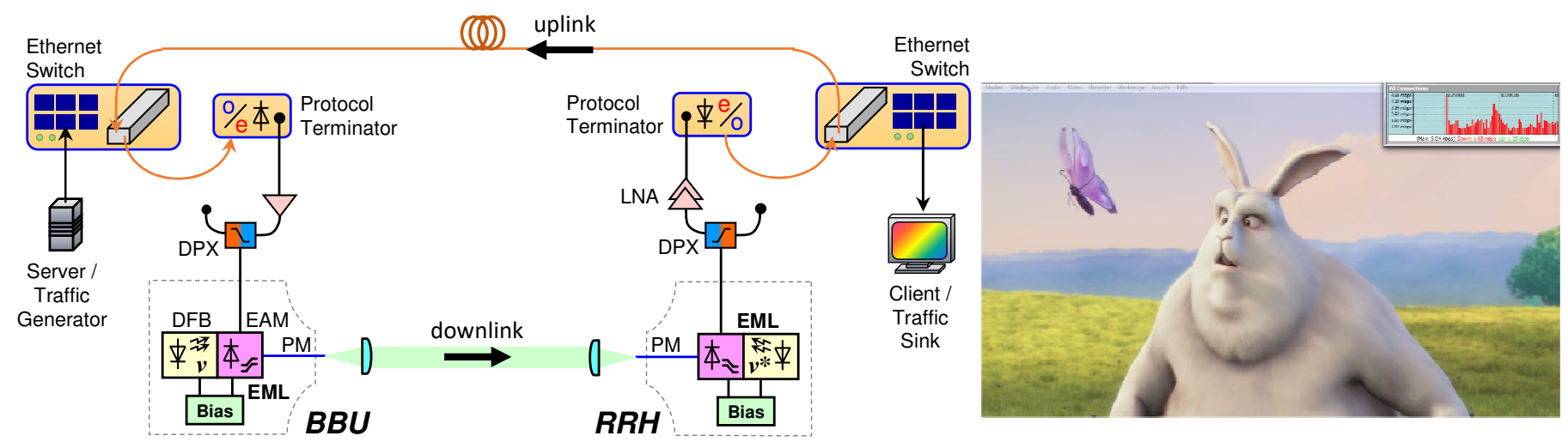

Fig. 9. Experimental setup to evaluate Ethernet-based payloads transmitted over the optical link. The inset shows a capture of a received video stream.

modulation extinction ratio of $8.6 \mathrm{~dB}$ is obtained. The average EVM drops with reduced bias for the receiving EML since crosstalk from direct-detection (DD) terms is reduced. It reaches an EVM value of $7.52 \%$ at a receiver bias of $-0.35 \mathrm{~V}$. A typical received RF spectrum for the uplink is shown in Fig. 5(b). When the DFB section is left unbiased, DD terms can be seen above the noise floor. Under DFB bias the optical signal reception benefits from the sensitivity gain of coherent detection, which lifts the signal clearly above the DD terms. However, these terms do render as non-negligible when comparing to the noise background. The early saturation in the detected signal magnitude when increasing the EAM bias [17] proves the operation at a low bias of $-0.35 \mathrm{~V}$ as feasible.

In view of full-duplex operation at a common bias value, the bias of the receiving EML was fixed to this optimal value of $-0.35 \mathrm{~V}$ and the bias of the transmitting EML was pulled down to the same level (curve $\tau$ ). Due to a reduced intensity modulation index of $4.2 \mathrm{~dB}$, the EVM increases to $7.9 \%$ as the transmitter bias falls to $-0.35 \mathrm{~V}$. However, the EVM remains below the limit of $8 \%$ [19]. It shall be stressed that the rather high reduction in modulation extinction ratio does not affect the performance of the radio signal in the same way as it would apply to two-level on-off keying. This is because of the non-linearity of the EAM, which introduces an EVM floor that increases with the optical modulation amplitude [20].

\section{EVM Performance}

After setting the optimal bias point, the full-duplex EVM performance has been investigated. The corresponding EVM measurements for full-duplex up- $\left(f_{\mathrm{U}}\right)$ and downlink $\left(f_{\mathrm{L}}\right)$ transmission are presented in Fig. 5(c) and 5(d), respectively.

The uplink at $f_{\mathrm{U}}(\boldsymbol{\Lambda})$ shows an average EVM of $7.21 \%$ for an optical loss budget of $19.3 \mathrm{~dB}$ between the two EML fiber ports, including the free-space optical path. The clear 64QAM constellation diagram for the compound OFDM signal evidences that coherent homodyne reception and full-duplex operation is supported, without resorting to additional DSP functions. Only a small fraction of 6 out of the 124 data subcarriers show an EVM value above the set limit of $8 \%$. For comparison with the full-duplex EML transceiver, a commonly used PIN/TIA receiver has been used for signal reception in half-duplex mode $(\bullet)$. This direct-detection receiver, which enjoys a noise-optimized TIA front-end and a better fiber-to-chip coupling efficiency, leads to an average EVM that is slightly improved by $0.73 \%$. Provided that the EML is enabling bidirectional signal transmission even with a sub-optimal LNA front-end at its receiver branch, this small penalty trades well in view of the cost-optimized full-duplex transceiver solution. The downlink EVM performance at $f_{\mathrm{L}}$ $(\diamond)$, which is reported in Fig. 5(d), shows an average of 7.46\% and performs marginally worse than the uplink. 

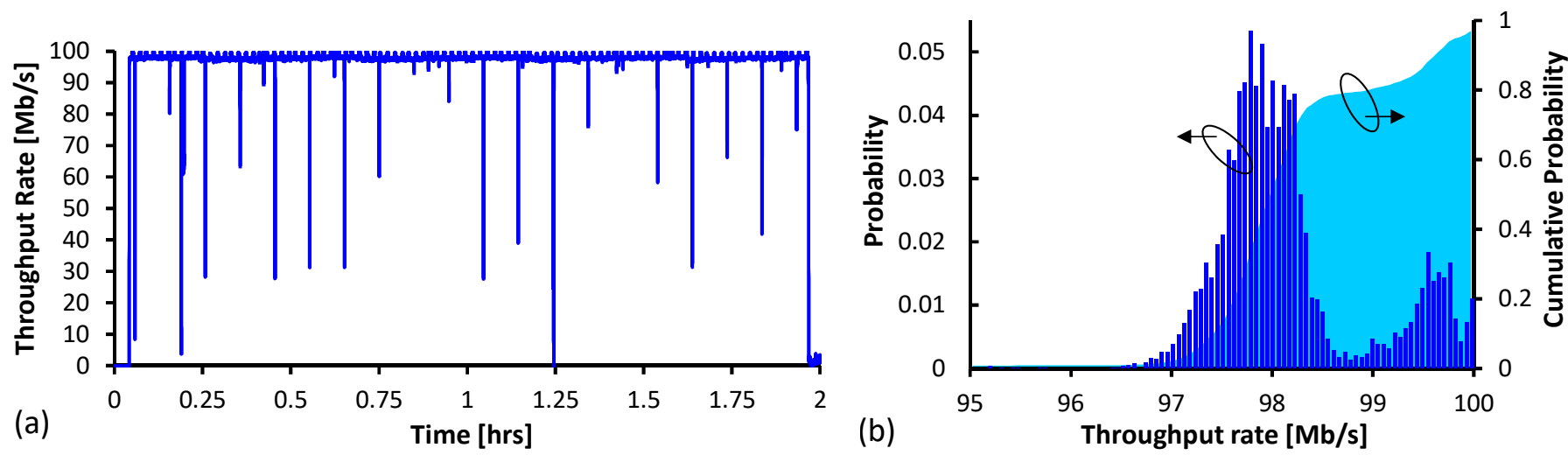

Fig. 10. Continuous long-term reception performance evaluation for Ethernet-based payloads. (a) Supported throughput rate and (b) corresponding histogram.

In order not to exclusively rely on EVM for the performance evaluation, the bit error ratio (BER) has been evaluated as an additional metric by means of bit error counting. Figure 6 presents the relation between the obtained EVM and the corresponding BER values. A low BER below typically used FEC thresholds can be obtained, which confirms the correctness of the EVM as an error metric. Moreover, the relation between BER and EVM stands in agreement with the model [21].

\section{Link Reach}

In order to roughly estimate the compatible link reach for the free-space optical fronthaul, the dependence of the average EVM on the optical loss budget between the EMLs has been investigated. Figure 7 presents the EVM as function of the optical loss budget for which ND filters have been inserted in the free-space path.

The compatible loss budget is $21.3 \mathrm{~dB}$ at the EVM limit of $8 \%$. The EVM improves marginally towards a lower loss budget, for which an error floor due to EAM non-linearity can be surmised. To estimate the corresponding free-space reach that is supported by this optical budget, geometric losses for the free-space link and fiber coupling efficiencies of 50\% have been taken into consideration. The geometric losses are derived from the transmitter and receiver aperture sizes and the beam divergence [22].

Figure 7 includes reach distances as function of the loss budget for two atmospheric scenarios: Firstly, an ideal channel that is not subject to further attenuation, which is referred to as "clear-sky" scenario (dashed line), and secondly, a worst-case scenario in which dense fog is assumed (chain-dotted line), leading to typical atmospheric attenuation of $110 \mathrm{~dB} / \mathrm{km}$ [22]. For this second scenario with unfavorable atmospheric conditions, a reach of $\sim 100$ meters can be anticipated for the compatible optical budget $(\Phi)$. This fits well to the deployment setting of a local C-RAN and falls in a range setting where additional atmospheric effects such as scintillations are negligible [23].

It shall be stressed that a practical deployment has to account for pointing, acquisition and tracking mechanism to account for thermal expansion or building sway for a fixed terrestrial installation. However, the complexity of these mechanisms can be greatly relaxed for stationary links by applying a wide enough beam at the transmitter in order to compensate for pointing errors due to the aforementioned effects [24].

Moreover, link margins should be considered to further account for vibrations and atmospheric turbulences [22]. Ambient noise due to scattered, reflected or direct sunlight requires filtering, which is partially accomplished through the coherent detection process. It shall be noted that the statistical incident probability for the most detrimental case of direct sunlight has been estimated with less than 1 hour per year [25].

\section{LINK STABILITY}

Long-term EVM measurements have been conducted for the uplink at $f_{\mathrm{U}}$ in order to investigate the stability of the faceto-face EML link architecture. Figure 8(a) presents the EVM per OFDM sub-carrier, which has been regularly monitored over a time period of more than one hour. The average EVM was $7.2 \%$ and only a few EVM excursions have been noticed. A fraction of $8.2 \%$ of all measurements is exceeding the EVM limit, as can be seen from the histogram in Fig. 8(b). These excursions are attributed to the cavity nature of the link, which is believed to result in sporadic mode hops and thus locking instabilities. However, for the majority of the measurement period, stable radio transmission is observed.

In order to allow for a more representative and continuous performance evaluation, an Ethernet connection has been collapsed over the downlink direction of the free-space optical link. The corresponding experimental setup is presented in Fig. 9. The 100M Ethernet signals of two switches have been fed to the EMLs at BBU and RRH site by means of protocol terminators, which serve as opto-electronic signal converter for the serial optical Ethernet signal. In this way an electrical representation of the Ethernet signal is yielded and can be transparently fed over the downlink channel of the EML-toEML link. For the sake of simplicity, the uplink return channel of the Ethernet link has been implemented as wired connection.

A server at the BBU site is used as traffic generator in order to load the Ethernet link. Moreover, it streams a highdefinition video with a resolution of $1280 \times 720$ pixels (sourced 
by Sample Video Developers). Both, the dummy traffic and the video are received and by a client that acts as sink for the purpose of continuous performance monitoring.

Figure 10(a) shows the Ethernet throughput over a duration of two hours, in which the received downlink traffic has measured in intervals of two seconds. The average rate for the loaded link is $97.9 \mathrm{Mb} / \mathrm{s}$. We have encountered 27 occurrences of reduced throughput, which indicate instabilities in the optical link. However, with a cumulated probability of $0.8 \%$ at a rate of $90 \mathrm{Mb} / \mathrm{s}$ (Fig. 10(b)), these excursions are rendered as very sporadic events. This confirms the link stability found in the earlier long-term EVM measurements. Moreover, the performance of the demonstrated link does align with the requirements sought for wireless fronthauling, for which availabilities of more than $99 \%$ are expected [26].

Finally, video streaming (see inset in Fig. 9) has been conducted in order to evaluate the impact on the sporadic excursions on real-time traffic, as they might trigger larger block errors. No artifacts have been noticed for the video transmission over a period of $15 \mathrm{~min}$, which confirms a steady end-to-end performance for the optical link.

\section{CONCLUSION}

We have experimentally demonstrated a conceptually simple, bidirectional free-space optical link based on a pair of EML transceivers. Full-duplex analogue 64-QAM OFDM radio transmission has been conducted in virtue of coherent homodyne detection, without resorting to DSP functions besides the mandatory OFDM demodulation. A small EVM penalty of $0.73 \%$ has been confirmed with respect to an ideal PIN/TIA receiver. A compatible link reach of $\sim 100 \mathrm{~m}$ has been estimated to be compatible, which stands in good agreement with local C-RAN applications based on a freespace optical fronthaul. Long-term measurements on EVM and Ethernet-based data transmission have confirmed the stability of the face-to-face EML link architecture. Only a small fraction of less than $1 \%$ of measurements showed an excursion, which confirms the principal stability of the optical link.

\section{ACKNOWLEDGMENT}

The authors would like to thank Mario Klima for his support during the Ethernet-based performance evaluation.

\section{REFERENCES}

[1] J. Wu, Z. Zhang, Y. Hong, and Y. Wen, "Cloud Radio Access Network (C-RAN): A Primer," IEEE Network, vol. 29, no. 1, pp. 35-41, Jan. 2015.

[2] Y. Mao, C. You, J. Zhang, K. Huang, K.B. Letaief, "A Survey on Mobile Edge Computing: The Communication Perspective," IEEE Communications Surveys \& Tutorials, vol. 19, no. 4, pp. 2322-2358, 2017.

[3] K. Sakaguchi et al., "Where, When, and How mmWave is Used in 5G and Beyond," IEICE Trans. Electron., vol. E100-C, no. 10, pp. 790-808, Oct. 2017.

[4] I.A. Alimi, A.L. Teixeira, P.P. Monteiro, "Toward an Efficient C-RAN Optical Fronthaul for the Future Networks: A Tutorial on Technologies, Requirements, Challenges, and Solutions," IEEE Communications Surveys \& Tutorials, vol. 20, no. 1, pp. 708-769, 2018
[5] T. Pfeiffer, "Next Generation Mobile Fronthaul and Midhaul Architectures," IEEE/OSA J. Opt. Comm. Netw., vol. 7, no. 11, pp. B38B45, Nov. 2015

[6] X. Liu, H. Zeng, N. Chand, and F. Effenberger, "Efficient Mobile Fronthaul via DSP-based Channel Aggregation," IEEE/OSA J. Lightwave Technol., vol. 34, no. 6, pp. 1556-1564, Mar. 2016.

[7] J. Yao, "Microwave Photonics," IEEE/OSA J. Lightwave Technol., vol. 27, no. 3, pp. 314-335, Feb. 2009.

[8] H.N. Parajuli, H. Shams, L. Guerrero Gonzalez, E. Udvary, C. Renaud, and J. Mitchell, "Experimental demonstration of multi-Gbps multi subbands FBMC transmission in mm-wave radio over a fiber system," OSA Opt. Expr., vol. 26, no. 6, pp. 7306-7312, Mar. 2018.

[9] J. Bohata, S. Zvanovec, T. Korinek, M. Mansour Abadi, and Z. Ghassemlooy, "Characterization of dual-polarization LTE radio over a free-space optical turbulence channel," OSA Appl. Opt., vol. 54, no. 23, pp. 7082-7087, 2015.

[10] D. Schulz et al., "Robust Optical Wireless Link for the Backhaul and Fronthaul of Small Radio Cells," IEEE/OSA J. Lightwave Technol., vol. 34, no. 6, pp. 1523-1532, Mar. 2016.

[11] R. Zhang et al., "An Ultra-Reliable MMW/FSO A-RoF System Based on Coordinated Mapping and Combining Technique for 5G and Beyond Mobile Fronthaul," IEEE/OSA J. Lightwave Technol., vol. 36, no. 20, pp. 4952-4959, Oct. 2018.

[12] B. Schrenk, D. Milovancev, N. Vokic, H. Hübel, and F. Karinou, "Radio-over-Air with a Face-to-Face EML Transceiver Pair", in Proc. Europ. Conf. Opt. Comm., Dublin, Ireland, Sep. 2019, Tu.3.C.2.

[13] B. Schrenk, and F. Karinou, "A Coherent Homodyne TO-Can Transceiver as Simple as an EML," IEEE/OSA J. Lightwave Technol., vol. 37, no. 2, pp. 555-561, Jan. 2019.

[14] N.H. Zhu et al., "Microwave generation in an electro-absorption modulator integrated with a DFB laser subject to optical injection," OSA Opt. Expr., vol. 17, no. 24, pp. 22114-22123, Nov. 2009.

[15] B. Schrenk, M. Hofer, and T. Zemen, "Analogue Receiver for Coherent Optical Analogue Radio-over-Fiber Transmission", OSA Opt. Lett., vol. 42, no. 16, pp. 3165-3168, Aug. 2017.

[16] S. Parkvall, E. Dahlman, A. Furuskär, and M. Frenne, "NR: The New 5G Radio Access Technology," IEEE Comm. Standards Mag., vol. 1, no. 4, pp. 24-30, Dec. 2017.

[17] B. Schrenk, "The EML as Analogue Radio-over-Fiber Transceiver - a Coherent Homodyne Approach," IEEE/OSA J. Lightwave Technol., vol. 37, no. 12, pp. 2866-2872, Jun. 2019.

[18] S.A. Busari, K.M.S. Huq, S. Mumtaz, L. Dai, and J. Rodriguez, "Millimeter-Wave Massive MIMO Communication for Future Wireless Systems: A Survey," IEEE Comm. Surveys \& Tutorials, vol. 20, no. 2, pp. 836-869, 2018.

[19] "LTE, Evolved Universal Terrestrial Radio Access (E-UTRA); Base Station (BS) radio transmission and reception," 3GPP, TS 36.104.

[20] B.G. Kim, S.H. Bae, H. Kim, and Y.C. Chung, "RoF-Based Mobile Fronthaul Networks Implemented by Using DML and EML for 5G Wireless Communication Systems," IEEE/OSA J. Lightwave Technol., vol. 36, no. 14, pp. 2874-2881, Jul. 2018.

[21] R.A. Shafik, M.S. Rahman, and A.R. Islam, "On the Extended Relationships Among EVM, BER and SNR as Performance Metrics," in Proc. Int. Conf. on Electr. and Comp. Eng., Dhaka, Bangladesh, pp. 408411, Dec. 2006.

[22] S. Bloom, E. Korevaar, J. Schuster, and H. Willebrand, "Understanding the performance of free-space optics," OSA J. Opt. Netw., vol. 2, no. 6, pp. 178-200, 2003.

[23] A. Prokes, "Atmospheric effects on availability of free space optics systems," SPIE Opt. Engineering, vol. 48, no. 6, p. 066001, Jun. 2009.

[24] Y. Kaymak, R. Rojas-Cessa, J. Feng, N. Ansari, M.C. Zhou, and T. Zhang, "A Survey on Acquisition, Tracking, and Pointing Mechanisms for Mobile Free-Space Optical Communications," IEEE Communications Surveys \& Tutorials, vol. 20, no. 2, pp. 1104-1123, 2018.

[25] M.A. Khalighi, and M. Uysal, "Survey on Free Space Optical Communication: A Communication Theory Perspective," IEEE Communications Surveys \& Tutorials, vol. 16, no. 4, pp. 2231-2258, 2014.

[26] J. Bartelt, P. Rost, D. Wübben, J. Lessmann, B. Melis, and G. Fettweis, "Fronthaul and Backhaul Requirements for Flexibly Centralized Radio Access Networks," IEEE Wireless Comm., vol. 22, no. 5, pp. 105-111, Oct. 2015 
Bernhard Schrenk (S'10-M'11) was born 1982 in Austria and received the M.Sc. ('07) degree in microelectronics from the Technical University of Vienna. He was at the Institute of Experimental Physics of Prof. A. Zeilinger, where he was involved in the realization of a first commercial prototype for a quantum cryptography system, within the European SECOQC project. From 2007 to early 2011 he obtained his Ph.D degree at UPC BarcelonaTech, Spain. His Ph.D thesis on multi-functional optical network units for nextgeneration Fiber-to-the-Home access networks was carried out within the FP7 SARDANA and EURO-FOS projects. In 2011 he joined the Photonic Communications Research Laboratory at NTUA, Athens, as post-doctoral researcher and established his research activities on coherent FTTH under the umbrella of the FP7 GALACTICO project. In 2013 he established his own research force on photonic communications at AIT Austrian Institute of Technology, Vienna, where he is working towards next-generation metroaccess-5G networks, photonics integration technologies and quantum optics.

Dr. Schrenk has authored and co-authored $~ 140$ publications in top-of-theline (IEEE, OSA) journals and presentations in the most prestigious and highly competitive optical fiber technology conferences. He was further awarded with the Photonics21 Student Innovation Award and the Euro-Fos Student Research Award for his PhD thesis, honoring not only his R\&D work but also its relevance for the photonics industry. He was elected as Board-ofStakeholder member of the Photonics21 European Technology Platform in 2017. During his extensive research activities he was and is still engaged in several European projects such as SARDANA, BONE, BOOM, APACHE, GALACTICO, EURO-FOS and the Quantum Flagship projects UNIQORN and CIVIQ. In 2013 he received the European Marie-Curie Integration Grant WARP-5. In 2018 he was awarded by the European Research Council with the ERC Starting Grant COYOTE, which envisions coherent optics everywhere.

Dinka Milovančev (M'19) was born in Serbia in 1990. She finished her M.Sc. studies in 2014 in Microelectronics from the University of Novi Sad, Serbia. She did her Ph.D. research at Vienna University of Technology in Austria at the Institute of Electrodynamics, Microwave and Circuit Engineering from 2014 up to 2019. Her Ph.D. research interests include circuit design of integrated avalanche photodiode receivers and their applications for optical wireless and fiber communication. Since 2019 she is employed at AIT Austrian Institute of technology, Vienna, where she works in the field of coherent optical fiber communication in classical as well as quantum optical domain.

Nemanja Vokić (M'19) was born in Serbia in 1990. He received the M.Sc. degree in electrical and computer engineering from the University of Novi Sad, Serbia in 2014. He obtained his Ph.D. degree from Vienna University of Technology in 2018. For his Ph.D. work, he designed electronic integrated circuits for transmitters and receivers used for optical communications. He was then a post-doc at Vienna University of Technology, designing mm-wave ASICs for 5G remote-radio-heads. In 2019, he joined AIT Austrian Institute of Technology, Vienna. His research interests include design of analog and
RF integrated circuits, opto-electronic circuit integration, coherent optical communication for telecom, datacom and quantum communications.

Hannes Hübel obtained his PhD in 2004 from Queen Mary, University of London, UK. In the same year he joined the quantum information group at the University of Vienna, headed by Anton Zeilinger, to work on experimental quantum communication, in particular Quantum Key Distribution (QKD). In 2008, he presented the first realization of a fully automated QKD system based on entanglement, within the European SECOQC project. He then worked as a post-doctoral researcher at the University of Waterloo, Canada, focusing on experimental demonstrations of multipartite entanglement. In 2010, he became assistant professor at the University of Stockholm, Sweden. Since 2015, he leads the experimental QKD development at the AIT Austrian Institute of Technology in Vienna, Austria.

Dr. Hübel has authored and co-authored $\sim 50$ publications in top-of-the-line journals and more than 30 invited and contributed presentations at international conferences. He builds on practical experience from the coordination of national (TransQ, QKD Telco, KVQ) and contracted industrial projects, as well as on experience in steering and participation in large European projects including QT-Flagship projects (QAP, SECOQC, UNIQORN, CIVIQ, OPENQKD).

Fotini Karinou (S'09-M'13) was born in Arta, Greece, in 1983. She received the Diploma in Electrical and Computer Engineering, with specialization in telecommunications and information theory, and the $\mathrm{PhD}$ degree in optical communications focused on spectrally efficient WDM optical interconnect networks with advanced modulation formats, from the University of Patras, Greece, in 2007, and 2012, respectively. In 2007, she was a visiting researcher in the Institute for Quantum Optics and Quantum Information (IQOQI), at Vienna University of Technology, participating in the initial attempts for the realization of a commercial prototype of a quantum key distribution (QKD) system based on polarization entanglement in the Institute of Experimental Physics of Prof. Zeilinger. In 2011 to 2012, she was a visiting researcher at Denmark Technical University, Photonics Department, where she worked toward spectrum-flexible cognitive optical networks, hybrid optical fiberwireless transmission and advanced modulation techniques for highperformance computing optical interconnects. From February 2013 to June 2018, Dr. Karinou worked as a Senior R\&D Engineer in the Optical Technology Department, later restructured to Optical \& Quantum Laboratory, at Huawei Technologies Duesseldorf $\mathrm{GmbH}$, in the German Research Center in Munich, toward next generation high-capacity coherent systems for long reach, high data-rate metro/access optical networks, optical interconnects for data centers and quantum communications. In July 2018 she joined the Systems and Networking Group at Microsoft Research Ltd in Cambridge, UK, where she is working toward developing optical technologies for next generation cloud computing systems and networks. She has authored and coauthored 70 publications in the highest prestigious optical communications technology journals and conferences. Dr. Karinou has been engaged in several European research projects such as EU FP6 SECOQC, EU FP7 ICT-BONE, EU FP7 CHRON, and H2020 ROAM projects. 